\title{
Modulatory Effects of Triphala and Manjistha Dietary Supplementation on Human Gut Microbiota: A Double-Blind, Randomized, Placebo-Controlled Pilot Study
}

\author{
Christine T. Peterson, PhD, Aunna Pourang, $\mathrm{MD}^{2}$ Simran Dhaliwal, BS, ${ }^{2}$ \\ Jordan N. Kohn, PhD, Sasha Uchitel, BS, ${ }^{4}$ Harjot Singh, BS, MPH, ${ }^{5}$ Paul J. Mills, PhD, \\ Scott N. Peterson, $\mathrm{PhD}^{6}$ and Raja K. Sivamani, MD, MS, $\mathrm{AP}^{2,7-10}$
}

\begin{abstract}
Objectives: Triphala (which contains Emblica officinalis, Terminalia bellerica, and Terminalia chebula) and manjistha (Rubia cordifolia), have received increased clinical attention. The aim of the study was to evaluate the effects of triphala, manjistha, or placebo dietary supplementation on gut microbiota as such studies in humans are lacking.

Design: This was a 4-week randomized, double-blind, placebo-controlled pilot trial.

Setting: This trial was conducted at the University of California Davis, Department of Dermatology.

Subjects: A total of 31 healthy human subjects were randomized to 3 groups.

Interventions: The 3 groups were instructed to take 2,000 $\mathrm{mg}$ of either triphala, manjistha or placebo daily for 4 weeks.

Outcome Measures: The impact of treatment on gut microbiota composition was evaluated following a 4-week dietary intervention by profiling fecal communities with 16S rRNA profiling in triphala $(n=9)$, manjistha $(n=9)$, or placebo $(n=11)$ treated subjects that completed the intervention.

Results: An average of 336 phylotypes were detected in each sample (range: 161 to 648). The analysis of gut microbiota in placebo control and herb-supplemented participants indicated that responses were highly personalized, and no taxa were uniformly altered by the medicinal herb supplementation protocol. Subjects in both treatment groups displayed a trend toward decreased Firmicutes to Bacteroidetes ratio and increased relative abundance of Akkermansia muciniphila. Both medicinal herb treatments reduced the relative abundance of Rikenellaceae, primarily reflecting changes in Alistipes spp.

Conclusions: Dietary supplementation with medicinal herbs altered fecal microbial communities. Despite the lack of a clear response signature, a group of bacterial taxa were identified that were more commonly altered in herb-supplemented participants compared to placebo controls. Clinicaltrials.gov identifier NCT03477825.

Keywords: microbiota, triphala, manjistha, Akkermansia muciniphila, prebiotic, Ayurveda

\footnotetext{
${ }^{1}$ Department of Family Medicine and Public Health, Center of Excellence for Research and Training in Integrative Health,

${ }^{2}$ Department of Dermatology, University of California Davis, Sacramento, CA, USA.

${ }^{3}$ Department of Psychiatry, University of California San Diego, La Jolla, CA, USA.

${ }^{4}$ Department of Biology, Washington University, St. Louis, MO, USA.

${ }^{5}$ Department of Naturopathic Medicine, Bastyr University San Diego, La Jolla, CA, USA.

${ }^{6}$ Tumor Microenvironment and Cancer Immunology Program, Sanford Burnham Prebys Medical Discovery Institute,

${ }^{7}$ Department of Biological Sciences, California State University, Sacramento, CA, USA.

${ }^{8}$ College of Medicine, California Northstate University, Elk Grove, CA, USA.

${ }^{9}$ Pacific Skin Institute, Sacramento, CA, USA.

${ }^{10}$ Zen Dermatology, Sacramento, CA, USA.
} University of California San Diego, School of Medicine, La Jolla, CA, USA. La Jolla, CA, USA.
\end{abstract}




\section{Introduction}

$\mathbf{T}$ RIPHALA, A WIDELY studied polyherbal formulation (common name: triphala), composed of the dried fruits of Emblica officinalis (common name: amalaki), Terminalia bellerica (common name: bibhitaki), and Terminalia chebula (common name: haritaki), as well as Rubia cordifolia (common name: manjistha), is receiving increased clinical attention due to a burgeoning body of research demonstrating therapeutic potential. Ayurveda, as well as integrative medicine practitioners, commonly prescribes these herbal medicines for a variety of gut- and skin-related conditions. However, insufficient data exist on the effects of triphala or manjistha on gut microbiota, and clinical investigations of these herbal medicines are lacking.

Triphala is enriched in diverse polyphenolic compounds such as tannins, gallic acid, ellagic acid, chebulinic acid, quercetin, luteolin, saponins, and anthraquinones. Recent studies have evaluated the biotransformation of polyphenolic compounds by gut microbiota that result in increased bioabsorption and/or bioactivity. ${ }^{1}$ For example, gut microbial biotransformation of chebulinic acid, which has been shown to have direct effects on COX-2 expression, may lead to subsequent prevention of oxidative damage., ${ }^{2,3}$ Triphala supplementation also increased glutathione and phospholipids in rat intestinal brush border cells while reducing myeloperoxidase and xanthine oxidase levels in the epithelium. ${ }^{4}$ Several studies have highlighted triphala-mediated anti-inflammatory effects that include decreased proinflammatory cytokine production. ${ }^{5-7}$

Numerous in vitro studies have evaluated the antimicrobial effects of triphala. ${ }^{8-11}$ Triphala extract demonstrated antibacterial activity against Escherichia coli, Klebsiella pneumoniae, and Salmonella typhi isolates from HIV infected patients. ${ }^{12}$ While these studies are biased toward pathogenic microbes such as E. coli, S. typhi, Staphylococcus aureus, Pseudomonas aeruginosa, and others, they suggest antibacterial effects of triphala on both gram-positive and gramnegative species with potential for further investigation.

$R$. cordifolia (common name: manjistha) has traditionally been used for skin health, stimulation of lymphatic flow, and blood disorders. ${ }^{13}$ Manjistha root and extracts contain a number of bioactive phytochemical constituents, including flavonoids, saponins, carboxylic acids, polyphenols, and anthraquinones (e.g., purpurin, mollugin, rubiadin, and munjistin), that possess strong antioxidant properties and stimulate production of antioxidant markers such as superoxide dismutase, catalase, and glutathione. ${ }^{14,15}$ Various in vitro and animal model studies have reported the antioxidant and antiinflammatory potential of manjistha. ${ }^{16-20}$

Several studies reported the antibacterial activity of phenolic constituents in manjistha in vitro. ${ }^{21-24}$ Manjistha methanol extract demonstrated activity against gram positive bacteria Bacillus spp., Micrococcus luteus, Mycobacterium luteum, and $S$. aureus, but not the gram negatives E. coli, Salmonella typhimurium, Shigella dysenteriae, Vibrio cholerae, and $K$. pneumoniae. ${ }^{21}$ In addition, ethanolic extracts of manjistha displayed antimicrobial activity toward Candida albicans and methicillin-resistant $S$. aureus. ${ }^{22}$ Anthraquinone compounds derived from manjistha have also demonstrated antimicrobial activity against pathogens such as E. coli and S. aureus. ${ }^{23}$
Clinical studies on the effects of either triphala or manjistha on the human gut microbiome are lacking. The authors hypothesized that triphala and manjistha consumption would alter the gut microbiota composition distinctly from that observed in the placebo control group and that both triphala and manjistha would exert differential effects on subsets of the gut microbiota. In this study the authors report an analysis of the fecal microbiota of subjects consuming triphala, manjistha, or placebo in a 4-week, randomized, double-blind placebo-controlled pilot clinical trial. Sequencing and analysis of the V3-V4 region of the 16S rRNA gene indicated that the microbiota-modulatory effects of triphala and manjistha were highly personalized; however, each herb preferentially enriched or reduced the relative abundance of specific bacterial species.

\section{Materials and Methods}

\section{Study participants and sample collection}

The University of California, Davis Institutional Review Board (IRB \#1174110) approved this study (http:// ClinicalTrials.gov Identifier: NCT03477825), and written informed consent was obtained from all subjects before enrollment. A total of 36 subjects were screened and 31 subjects assigned to one of three groups, namely placebo, triphala, or manjistha treatment. The study demographics were as follows: Placebo: $37.2 \pm 11.6$ years of age ( 9 females, 2 males); Triphala: $33.0 \pm 8.7$ years of age ( 6 females, 4 males); and Manjistha: $30.7 \pm 9.7$ years of age ( 7 females, 3 males).

\section{Study intervention}

The Consort diagram outlines the treatment groups (Fig. 1). The manjistha (R. cordifolia), triphala (E. officinalis, T. bellerica, and $T$. chebula), and placebo tablets (containing microcrystalline cellulose, dicalcium phosphate, PVPK30, sodium starch glycolate, magnesium stearate, and Opadry orange coating) were provided by Banyan Botanicals (Ashland, OR). All groups were directed to take four tablets daily, which was a total of $2000 \mathrm{mg}$ in both the manjistha and triphala supplementation groups.

\section{Study design}

This study was designed as a placebo-controlled, randomized double-blinded study. All of the investigational products were provided in sealed and coded containers, and treatment allocation was performed a priori in sealed envelopes. Participants were excluded if they had any smoking history within the past year, history of diabetes, malignancy, known cardiovascular disease, known kidney disease, diagnosed metabolic syndrome, or had chronic steroid use. Those who had exposure to systemic antibiotics, probiotics, or any triphala or manjistha containing supplements within 1 month of participation were excluded. Pregnant and breastfeeding women were excluded as were those with a known allergy to $R$. cordifolia, E. officinalis, T. bellerica, or T. chebula.

\section{Study interventions and measures}

Participants were evaluated at baseline and 4 weeks postsupplementation by the study investigators. Participants 


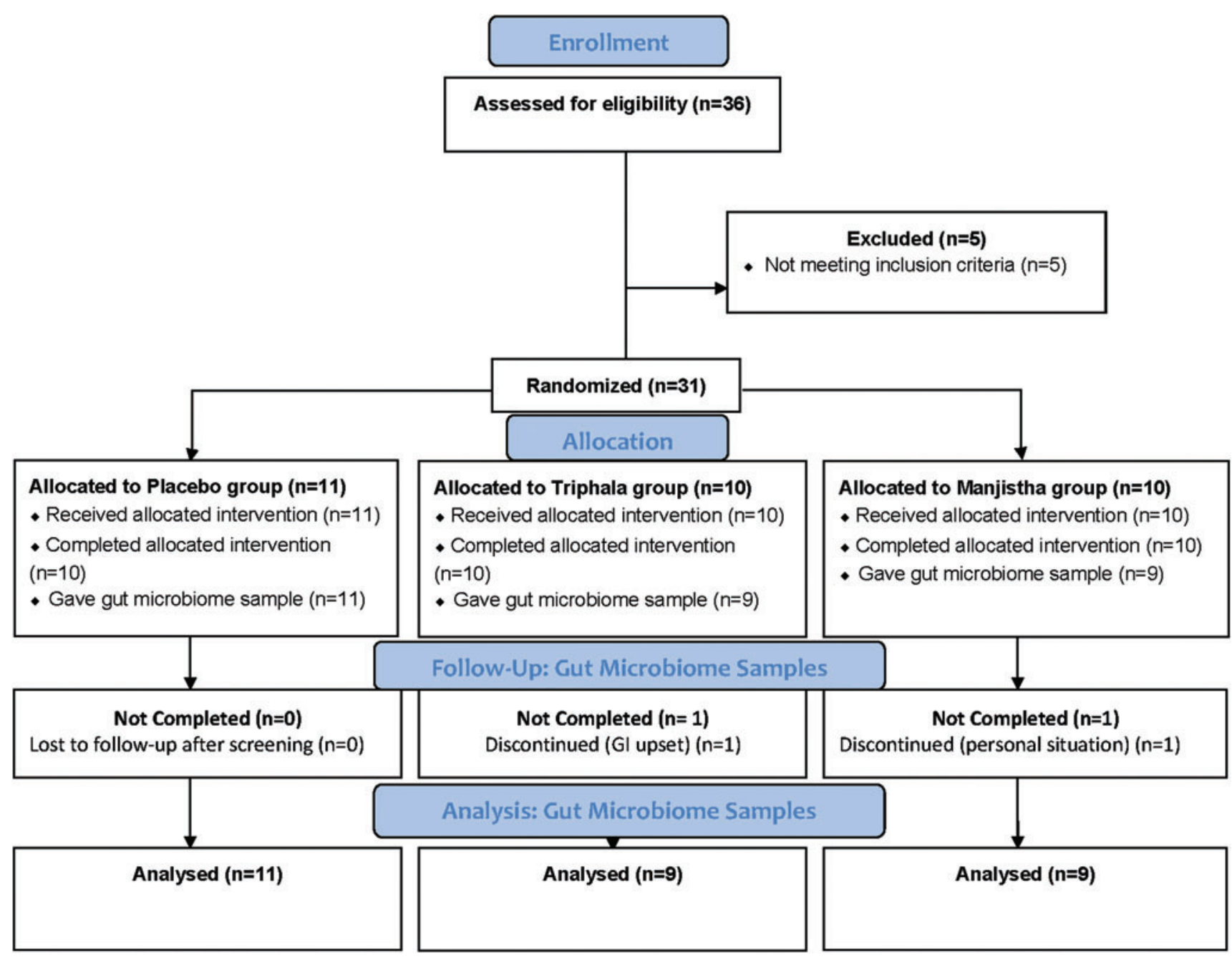

FIG. 1. Consort diagram. Color images are available online.

ate their normal (omnivorous) diets and donated a morning fecal sample in stool hats. The fecal samples were transferred to conical tubes and then frozen on ice and stored at $-80^{\circ} \mathrm{C}$ until further processing. Subjects were asked to report any adverse effects throughout the study.

\section{Microbial DNA isolation}

Genomic DNA was isolated from fecal samples using the procedures of the QIAamp DNA Stool Kit (Qiagen) with a modification that included an additional step of bead beating using the Thermo FastPrep instrument (MP Bio) to ensure uniform lysis of bacterial cells.

\section{$16 S$ rRNA sequence analysis}

Multiplexed 16S rDNA libraries were prepared using standard 16S metagenomic sequencing library protocols from Illumina, which uses V3-V4 region of 16S rDNA for target amplification, and subsequent analysis performed in CLC Microbial Genomics Module 2.5 (Qiagen) and $\mathrm{R}^{25}$ as the authors have described previously. ${ }^{26-28}$

\section{Statistical analyses}

Raw reads from 307 unique taxa were rarefied to the smallest depth across all samples (4976 reads) to adjust for differences in read depth for downstream differential abundance analysis. Low prevalence taxa ( $<10 \%$ of samples) were filtered out, and nonparametric aligned rank transform analysis of variance (ANOVA) was performed on abundances of the remaining 198 taxa using ARTool. ${ }^{29}$ Models were specified to test the interaction between treatment group (i.e., placebo, Triphala, or Manjistha) and visit (i.e., pre vs. postintervention), with random effect of participant to adjust for repeated within-subjects measurement. Type III Wald F-statistics and $p$-values were calculated using Kenward-Roger degrees of freedom for the interaction term, and $p$-values were adjusted for multiple comparisons (i.e., false discovery rate [FDR]) using BenjaminiHochberg correction. Taxa with FDR-corrected interaction term $p<0.05$ were retained, and post hoc $t$ tests for significant estimated marginal mean differences contrasting pre- and posttreatment visits within treatment groups were performed using emmeans, ${ }^{30}$ applying additional FDR-correction to adjust family-wise error rate. Contrasts were deemed significant at FDR-corrected $p<0.05$. Calculation of microbial 
diversity metrics, Shannon, Simpson, and Inverse Simpson, was performed using the vegan package in R. Plots were generated using ggplot 2 in $\mathrm{R}$.

\section{Results}

The authors assessed the fecal microbiota of subjects pre and post a 4-week intervention with placebo $(n=11)$ or the medicinal herbs triphala $(n=9)$ or manjistha $(n=9)$. The authors sequenced the V3-V4 region of the 16S rRNA gene to enumerate species present in participant stool samples. A total of 4,655,234 clones were sequenced ( mean $=80,236 \pm 15,719 ;$ lowest $=4976$ reads $/$ sample $)$.

In total, the authors observed 1318 phylotypes, defined as unique $16 \mathrm{~S}$ rRNA sequence differing by as little as 1 base position. Among these phylotypes, 243 were observed in 50\% of human subject participants, and 89 were present in $80 \%$ of participants (Fig. 2A). The average number of phylotypes observed in paired stool samples (pre and post) was comparable (ave $=446$, range $=271-664$ ) for each group. Similarly, the number of phylotypes displaying altered relative abundance was consistent across all participants independent of their group (Fig. 2B). The authors analyzed the phylum present in placebo controls compared to herb-treated subjects and noted substantial variability across subjects (Fig. 3A). A comparison of average relative abundance of phyla indicated that subjects supplemented with either herb underwent a shift in their microbiota that reduced the Firmicutes to Bacteroides ratio (Fig. 3B) as the result of increased relative abundance of Bacteroidetes and reduced representation of Firmicutes. The relative abundance of Verrucomicrobia, due to the species Akkermansia muciniphila, was increased in
FIG. 2. Phylotype analysis. (A) The frequency of 1318 phylotypes observed in 58 stool samples was calculated to determine those that were prevalent in the human cohort. (B) The average number of phylotypes observed, increased in relative abundance ( $>5$-fold) or decreased relative abundance ( $>5$-fold), was calculated for each paired stool sample and averaged for each group. Color images are available online.
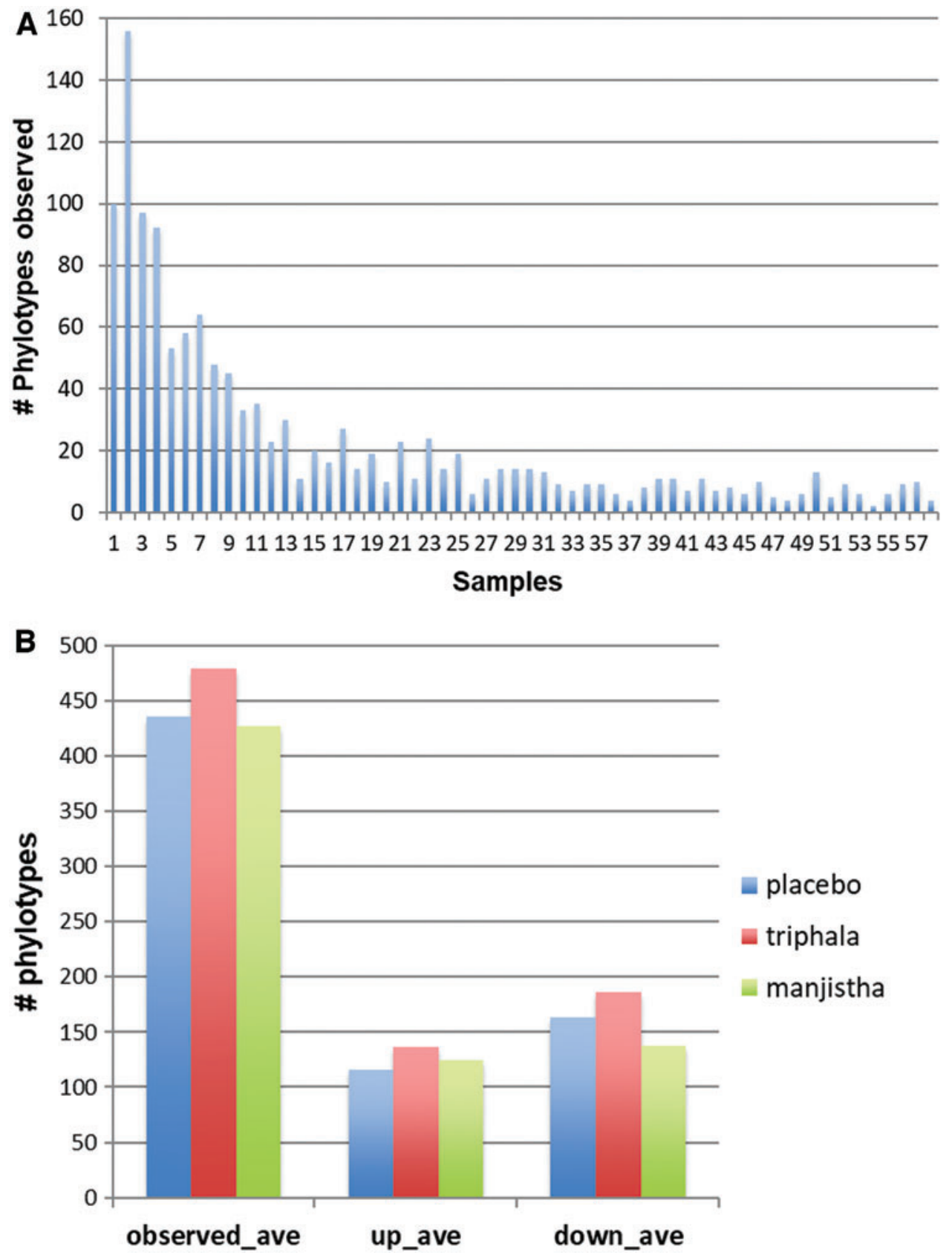


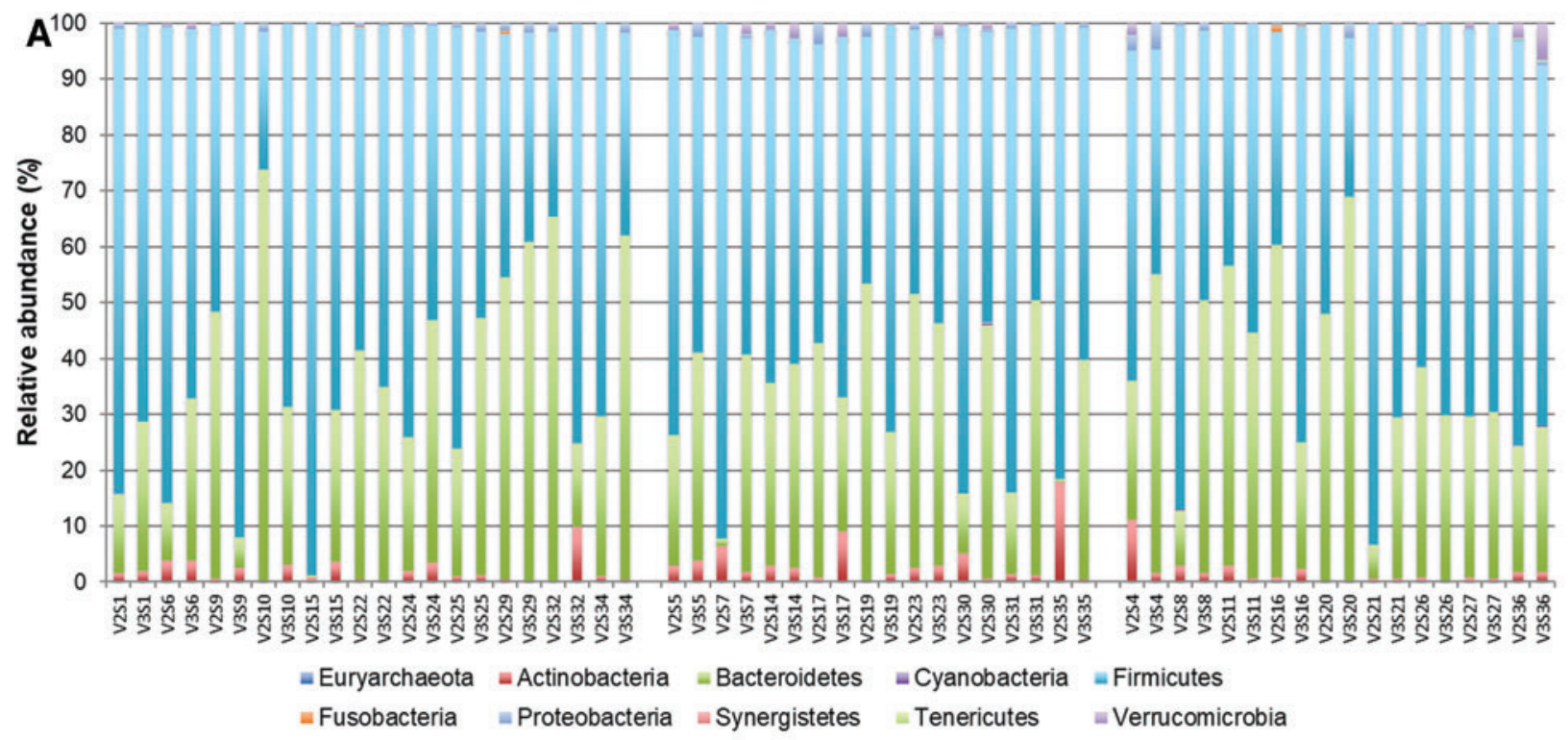

B

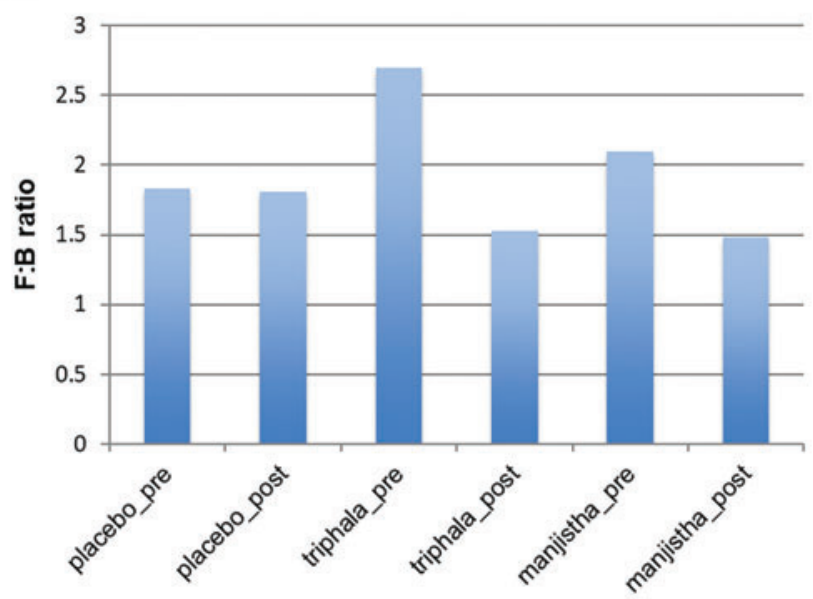

C

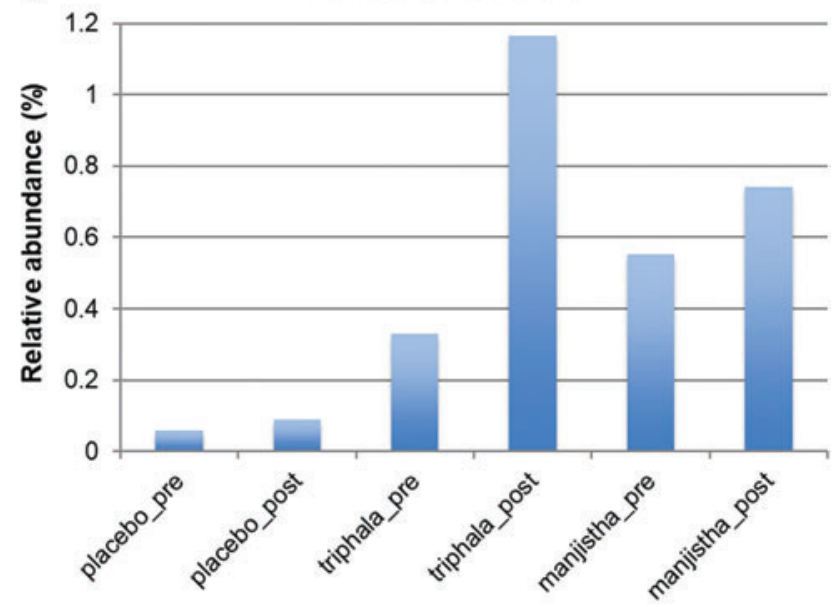

FIG. 3. Phylum-level analyses. (A) Distribution of the relative abundance of phyla observed in each subject, placebo control (left), triphala (center), manjistha (right). (B) The ratio of Firmicutes to Bacteroidetes depicted as an average for each study group. (C) The relative abundance of the phylum Verrucomicrobia depicted as an average for each study group. Color images are available online.

both herb-treated groups and particularly evident in triphala subjects (Fig. 3C). None of these differences reached statistical significance by $t$ test, due to high subject-to-subject variability within each treatment group.

\section{Herb-induced microbiota restructuring of bacterial families}

Given the high microbiota composition intersubject variability, the authors evaluated various taxonomic levels with respect to the frequency with which taxa responded positively or negatively to herb supplementation compared to placebo controls. The triphala group displayed a disproportionate increase in relative abundance of eight families, including Prevotellaceae, Barnesiellaceae, Enterococcaceae, Peptostreptococcaceae, Alcaligenaceae, Verrucomicrobiaceae, Streptococcaceae, and Christensenellaceae (Supple- mentary Table S1). Disproportionate taxa were defined as displaying concordant behavior (either increased or decreased) at a frequency of at least two instances more than placebo control subjects. The significance of these differences may be prone to error and should be interpreted with caution; however, five of these families displayed differential increases in three more subjects in the triphala group, and Prevotellaceae increases were observed at a differential frequency of 4 . The effects of manjistha were more modest with an increase in the proportion of subjects displaying increased relative abundance of just two families: Alcaligenaceae and Enterococcaceae, the first being differentially increased in three more instances compared to placebo control. Conversely, triphala supplementation resulted in an increased frequency of subjects displaying reduced relative abundance of five families, including Bacteroidaceae, $\mathrm{Ru}$ minococcaceae, Erysipelotrichaceae, Coriobacteriaceae, and 
Rikenellaceae; none of these families exceeded a differential occurrence $>2$, whereas manjistha disproportionately displayed reduced relative abundance of only Rikenellaceae that occurred in three more subjects compared to placebo control.

Among the 152 genera profiled, several were altered by herb supplementation compared to placebo controls with respect to the frequency of treatment-responsive subjects. The impacted genera were consistent with family-level results in many instances. In total, triphala supplemented subjects displayed an increased proportion of 26 genera displaying increased relative abundance in 2 more instances compared to controls, 11 of which displayed a differential of 3 and 4 genera with a differential increase in 4 instances. A total of 13 genera displayed decreased relative abundance compared to placebo control subjects, 3 of which were under-represented in 3 more subjects compared to placebo controls (Supplementary Table S2A). The genera increased and define a consortium with functionalities enriched in glycosyl hydrolase $(\mathrm{GH})$ gene loci that catabolize herb glycans that the authors have previously reported to be strongly influenced by glycan content of medicinal herbs. ${ }^{26-28}$ Among the taxa displaying increased abundance following triphala supplementation, Prevotella, Akkermansia, Bacteroides, and Barnesiella encode numerous GH specificities. The potential functional redundancy of the GH specificities may account for the variability of taxa induced across subjects. The liberation of oligo-, di-, and mono-saccharides by these taxa cross-feeds a number of fermentative taxa, including Colidextribacter, Intestinimonas, Ruminiclostridium, Anaerobacterium, Eisenbergiella, Gemmiger, Lachnobacterium, Lachnoclostridium, Pseudoflavonifractor, and Roseburia species. Taken together, these taxa displayed increased relative abundance in $16 \%$ of subjects in the placebo control group compared to $59 \%$ in the triphala group. In total, 12 genera were decreased in a disproportionate number of subjects following triphala supplementation. These taxa displayed decreased relative abundance in $18 \%$ of subjects in the placebo control group compared to $52 \%$ in the triphala group.

Similar to family level analysis, manjistha supplementation altered fewer genera, with six genera displaying an increase in proportion in subjects displaying increased relative abundance and eight genera displaying reduced relative abundance (Supplementary Table S2B). The functional relationship of these genera is not evident and did not define a food web as observed for triphala.

Among the 1318 phylotypes profiled the authors were able to assign these to 161 species sharing $>99 \%$ sequence identity to named human isolates. Consistent with analyses of higher taxonomic groups, the authors noted that herbal supplementation altered gut microbiota in an individual manner that were nonuniform across subjects, and therefore, no herb-specific signature was identified for triphala or manjistha responses. However, each herbal medicine did increase or decrease taxa in a disproportionate number of cases in treated subjects compared to placebo controls. In total, a higher frequency of subjects in the triphala group displayed an increase in the relative abundance of 47 species, 8 of which displayed differential increases of 3 and 7 displaying differential increases of 4 instances compared to controls. The authors noted decreased relative abundance of 29 species only, 4 of which were observed in 3 instances more compared with placebo controls (Supplementary
Table S3A). Subjects in the manjistha group displayed a disproportionate increase in the relative abundance of 25 species and a decrease in relative abundance of 19 species (Supplementary Table S3B).

Triphala increased the relative abundance of six Clostridium spp., three Bacteroides spp., and three Eubacterium spp. In addition, triphala increased the relative abundance of two Citrobacter spp., Coprococcus spp., Lactobacillus spp., Prevotella spp., and Roseburia spp. Triphala decreased the relative abundance of five Clostridium spp., three $R u$ minococcus spp., two Alistipes spp., Blautia spp., and Eisenbergiella spp. (Supplementary Table S3A). Manjistha increased the relative abundance of seven Clostridium spp. and two Lactobacillus spp. Unexpectedly, none of these species belong to those encoding large numbers of $\mathrm{GH}$ genes. Indeed, manjistha increased the proportion of subjects displaying decreased relative abundance of three Bacteroides spp. and Parabacteroides merdae (Supplementary Table S3B).

While the study was underpowered to formally test for and detect group differences between pre- and posttreatment samples, all three indices of alpha diversity (e.g., Shannon, Simpson, and inverse Simpson) indicated increased alpha diversity in the triphala group compared to the manjistha group. The authors calculated alpha diversity and plotted those results (Fig. 4 and Supplementary Table S4). While the study is underpowered to detect treatment $\mathrm{x}$ visit interactions for alpha diversity, there is an emergent trend toward increased alpha diversity in only the triphala group (Supplementary Table S5). Differential abundance analysis revealed 18 differentially abundant species (out of 198) at FDR $<0.05$ (Fig. 5and Supplementary Table S6).

Many but not all of the differentially abundant taxa were also altered in the placebo control group emphasizing the high level of dynamic change in all groups over time.

\section{Adverse events}

Each subject reported adherence to the treatment protocol, and no adverse effects were reported.

\section{Discussion}

Several studies of gut microbiota response to polyphenolic compounds demonstrate their modulatory effects on community composition; however, these prebiotic effects are likely to be indirect resulting from dampened host inflammation or oxidative stress. ${ }^{1}$ Their previous study examined the effect of triphala on fecal communities in vitro, suggesting that amino acids and complex carbohydrate components of triphala drive strong compositional changes, ${ }^{26}$ thus highlighting the complexity of the impact of herbs on gut microbiota using both direct and indirect mechanisms and in turn the potential importance of herbal effects being based, in part, on the metabolic capacity of gut microbiota. In addition, their results support the notion that herbs can have a prebiotic influence on the gut microbiota. ${ }^{26-28,31}$

Previously, the authors reported that human subjects supplemented with either turmeric or curcumin resulted in a personalized response defined by a common set of taxa altered by herb in some but not all subjects evaluated. ${ }^{31}$ In the current pilot clinical trial, the authors studied the impact of triphala and manjistha $(2 \mathrm{~g} /$ day $)$ supplementation in 

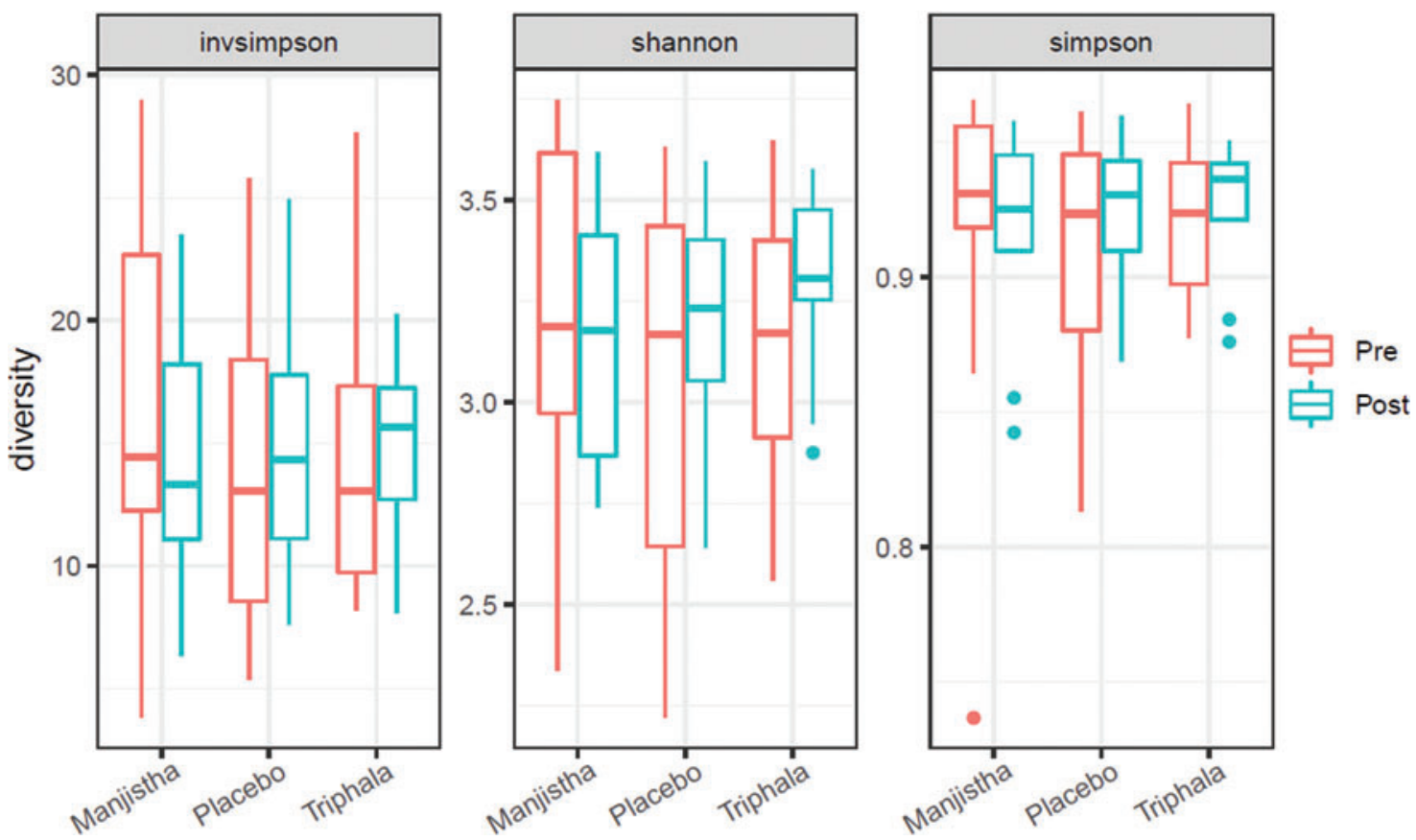

FIG. 4. Community diversity analysis. Boxplots of alpha diversity metrics, Shannon, Simpson, and Inverse Simpson diversity across treatment groups and study visits (pre- vs. post-treatment samples). Red bars and points indicate pretreatment values; blue bars and points indicate post-treatment values. Color images are available online.

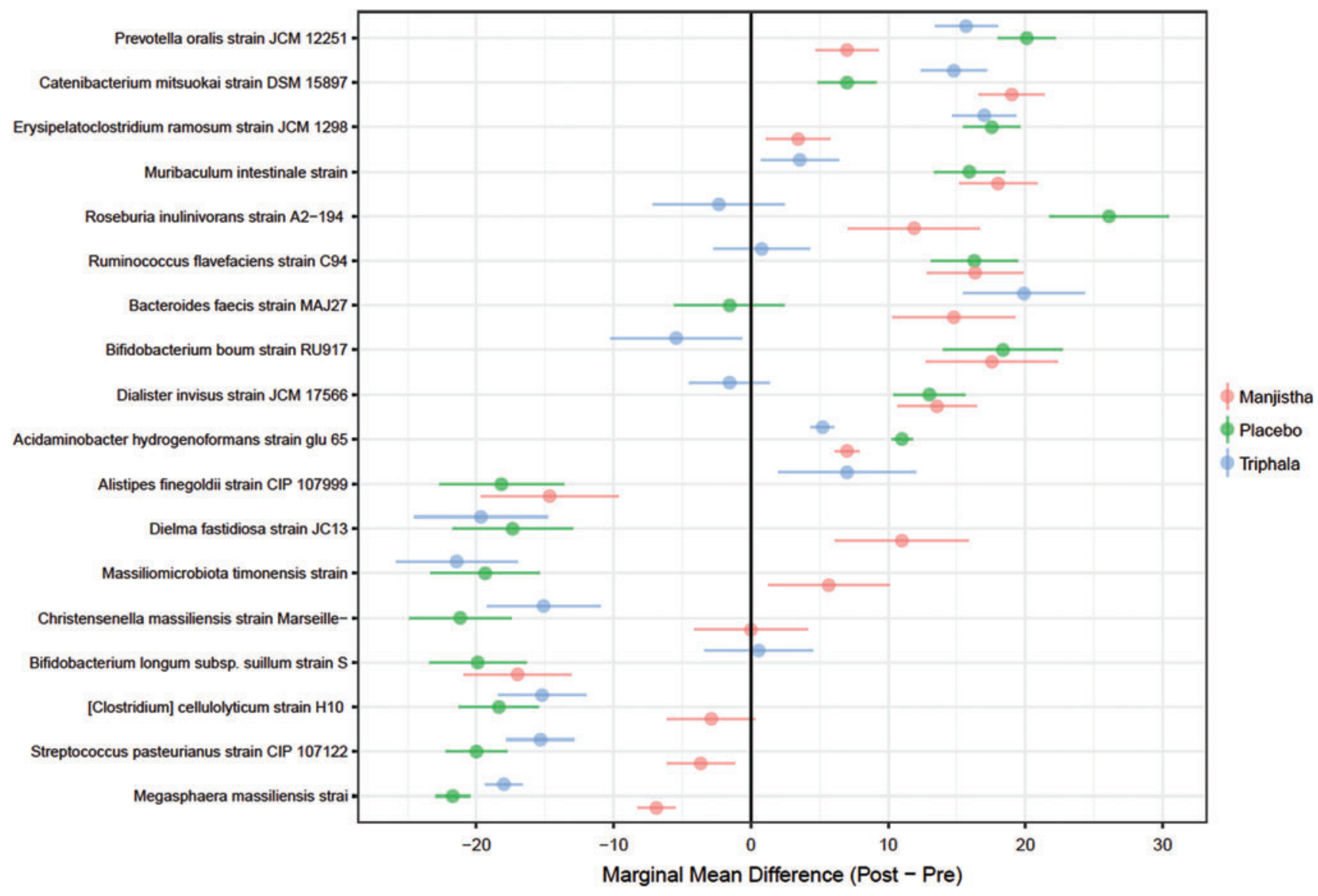

FIG. 5. Forest plot of estimated marginal mean differences. Marginal means were computed for differentially abundant microbial species, as determined by aligned-rank transformation ANOVA, comparing pre-to-post changes in relative abundance. Values to the left of vertical " 0 " reflect decreases in relative abundance, whereas values to the right indicate increases in relative abundance. Group means and 95\% confidence intervals shown. ANOVA, analysis of variance. Color images are available online. 
a 4-week intervention of healthy human subjects to analyze the impact of these medicinal herbs on gut microbiota composition. In contrast to observations made with turmeric and curcumin interventions, neither triphala nor manjistha induced alterations in fecal microbiota that were reproducible across subjects within the treatment groups. Analysis of the placebo group indicated that multiple taxa underwent large changes over the duration of the study making it challenging to identify taxa that were uniquely altered by herb treatment using standard statistical comparisons. To identify taxa that may be altered by triphala and manjistha, the authors applied a test to reveal taxa that were disproportionately increased or decreased in herbsupplemented groups compared to placebo controls. The disproportionately altered taxa may be associated with a false discovery rate suggesting that these taxa be interpreted cautiously and considered candidates requiring further testing and validation.

Despite the high intersubject variability in microbiota profiles at baseline and at the end of the 4-week intervention certain alterations were evident. The authors found that the average relative abundance of Firmicutes and Bacteroidetes was altered to reduce the average $F$ :B ratio in each herb treatment group compared to placebo control. The F:B ratio is indicative of phylum level changes encompassing numerous species comprising those phyla. It has been reported by several groups that the F:B ratio of lean subjects is reduced in lean compared to obese subjects in crosssectional studies of microbiota and obesity ${ }^{32}$ and animal models comparing low and high fat chow. ${ }^{33}$ Triphala consumption has been associated with reduced fat mass, cholesterol, and triglycerides suggesting that the herb-induced change in $\mathrm{F}: \mathrm{B}$ ratio represents a response signature of these herbs. These results are consistent with other studies that demonstrated that the abundance of A. muciniphila is negatively correlated with BMI and more abundant in lean subjects. ${ }^{34,35}$ Herb treatment groups, particularly the triphala group, displayed an increase in Verrucomicrobia, reflecting its only representative in gut microbiota, $A$. $m u$ ciniphila. Both triphala and manjistha reduced the relative abundance of Rikenellaceae, primarily reflecting the relative abundance of Alistipes spp. that are propionate and indole producing microbes. One study evaluated the correlation of sulfonolipids and weight gain resulting from high fat diet. Analysis of metagenomic sequence revealed that Alistipes spp. encode functions involved in sulfonolipid biosynthesis. $^{36}$ Additional studies show that Alistipes among other taxa were induced by an animal-based diet and are bile acid tolerant. ${ }^{37}$

Analysis of additional taxonomic levels revealed consistent results in terms of taxa that were disproportionately increased by herb supplementation compared to placebo control. Analyses at the family level revealed multiple families that were disproportionately altered by herb supplementation; the results were largely supported at the genus and species level demonstrating the self-consistency of analyses. These results suggest that family and genus-level changes observed are not due to small coordinated changes of numerous species, but rather due to larger changes in abundance of a small number of species representing each genus and family. It is likely that the taxa identified in this study reflect only a subset of species that were responsive to herb supplementation. The high inter- and intrasubject variability of placebo control subjects over time suggests that factors such as diet, genetics, and lifestyle may largely be dominant thereby masking potential herb-related effects. Interestingly, analysis of phylotypes (not shown), approximating strains and highly related species, indicated that in no case did all phylotypes belonging to particular species groups behave identically in response to herb supplementation, suggesting that herb responsiveness is not a trait encoded in the core genome of responsive species. This finding is consistent with their previously published work examining herb responses in vitro. ${ }^{26-28}$

The pilot scale data analyzed here support that herb supplementation stimulated alterations in fecal microbial communities. The clarity of the modulatory effects of triphala and manjistha was hampered by the high inter- and intrapersonal variation in gut microbiota longitudinally. These results contrast their previous observations pertaining to gut microbiota alterations induced by turmeric and curcumin dietary intervention, where substantial uniformity in taxa-specific changes across herb-responsive subjects was documented. ${ }^{31}$ The precise reason for the differing outcomes of the two trials may be instructive. The small number of participants, short duration of the intervention, and dosing may have contributed to the results obtained. The authors further speculate that turmeric and curcumin exerted a dominant effect with respect to diet and other environmental factors to drive coherent changes in responsive subjects that were not evident in nonresponsive subjects.

The dominance/recessive relationships of medicinal herbs compared to other environmental factors (e.g., diet) are likely to vary for any medicinal herb and dictate the clarity with which herb-specific effects on gut microbiota can be defined. The lack of a congruent set of triphala and manjistha-responsive taxa may reflect a greater flexibility in microbial configurations adopted in response to herb supplementation, thereby presenting functional coherence that is independent of taxonomic coherence. Such a functional signature was revealed in the triphala group where consortia include taxa encoding diverse glycan catabolism potential and fermentative species that cross feed on liberated sugar substrates. In this regard, future studies may benefit from shotgun metagenomic sequencing and/or RNA-Seq of fecal communities to evaluate functional relatedness across herb-responsive subjects and to extend the analyses beyond bacteria. In addition, future studies should connect the findings here with other readouts such as short chain fatty acids. Ultimately, these pilot studies provide important information to guide planning of larger efforts and importantly to establish cohorts of the appropriate size.

\section{Data Availability}

The 16S rRNA sequence data have been deposited at NCBI: SUB7435384 Bioproject PRJNA633107 Accessions SRR11797833-SRR1179890.

\section{Ethical Approval}

The University of California, Davis Institutional Review Board (IRB \#1174110) approved this study, and written 
informed consent was obtained from all subjects before enrollment.

\section{Authors' Contributions}

R.K.S., A.P., and S.D. designed and led the study intervention. R.K.S., A.P., and S.D. oversaw the study, led the clinical interventions, and supervised data/sample collection. C.T.P. designed the microbiome study. C.T.P. wrote the article, revised the article, and performed biologic interpretation. C.T.P., S.N.P., H.S., and S.U. performed genomics assays, quality control, and generated figures. C.T.P. analyzed the microbiome data. J.N.K. performed statistical analyses. R.K.S. supervised the study overall. All authors were also involved with biologic interpretation, article writing, and article revisions. All authors reviewed the article, revised the article, and approved of the final version for publication.

\section{Author Disclosure Statement}

The authors declared the following potential conflicts of interest with respect to the research, authorship, and/or publication of this article: R.K.S. has no relevant conflicts of interest and serves as a scientific advisor for LearnHealth and Arbonne and as a consultant for Burt's Bees, Regeneron, and Abbvie. P.J.M. is Director of Research for the Chopra Foundation. The other authors have no conflicts of interest to declare.

\section{Funding Information}

The authors disclosed receipt of the following financial support for the research, authorship, and/or publication of this article: This work was supported by departmental funding from the Department of Dermatology at UC Davis (R.K.S.) and a fellowship grant from the Chopra Foundation (C.T.P.).

\section{Supplementary Material}

Supplementary Table S1

Supplementary Table S2

Supplementary Table S3

Supplementary Table S4

Supplementary Table S5

Supplementary Table S6

\section{References}

1. Rowland I, Gibson G, Heinken A, et al. Gut microbiota functions: Metabolism of nutrients and other food components. Eur J Nutr 2018;57:1-24.

2. Reddy DB, Reddy TC, Jyotsna G, et al. Chebulagic acid, a COX-LOX dual inhibitor isolated from the fruits of Terminalia chebula Retz., induces apoptosis in COLO-205 cell line. J Ethnopharmacol 2009;124:506-512.

3. Olennikov DN, Kashchenko NI, Chirikova NK. In vitro bioaccessibility, human gut microbiota metabolites and hepatoprotective potential of chebulic ellagitannins: A case of Padma Hepaten(R) Formulation. Nutrients 2015;7:8456-8477.

4. Nariya MB, Shukla VJ, Ravishankar B, Jain SM. Comparison of gastroprotective effects of triphala formulations on stress-induced ulcer in rats. Indian J Pharm Sci 2011;73: 682-687.
5. Kalaiselvan S, Rasool MK. The anti-inflammatory effect of triphala in arthritic-induced rats. Pharm Biol 2015;53:51-60.

6. Kalaiselvan S, Rasool M. Triphala exhibits anti-arthritic effect by ameliorating bone and cartilage degradation in adjuvant-induced arthritic rats. Immunol Invest 2015;44: 411-426.

7. Kalaiselvan S, Rasool MK. Triphala herbal extract suppresses inflammatory responses in LPS-stimulated RAW 264.7 macrophages and adjuvant-induced arthritic rats via inhibition of NF-kappaB pathway. J Immunotoxicol 2016; 13:509-525.

8. Biradar YS, Jagatap S, Khandelwal KR, Singhania SS. Exploring of antimicrobial activity of Triphala Mashi-an Ayurvedic Formulation. Evid Based Complement Alternat Med 2008;5:107-113.

9. Tambekar DH, Dahikar SB. Antibacterial activity of some Indian Ayurvedic preparations against enteric bacterial pathogens. J Adv Pharm Technol Res 2011;2:24-29.

10. Srinagesh J, Krishnappa P, Somanna SN. Antibacterial efficacy of triphala against oral streptococci: An in vivo study. Indian J Dent Res 2012;23:696.

11. Shanbhag VK. Triphala in prevention of dental caries and as an antimicrobial in oral cavity-A review. Infect Disord Drug Targets 2015;15:89-97.

12. Srikumar R, Parthasarathy NJ, Shankar EM, et al. Evaluation of the growth inhibitory activities of Triphala against common bacterial isolates from HIV infected patients. Phytother Res 2007;21:476-480.

13. Shan M, Yu S, Yan H, et al. A review of the botany, phytochemistry, pharmacology and toxicology of Rubiae Radix et Rhizoma. Molecules 2016;21:1747.

14. Shilpa PN, Venkatabalasubramanian S, Devaraj SN. Ameliorative effect of methanol extract of Rubia cordifolia in N-nitrosodiethylamine-induced hepatocellular carcinoma. Pharm Biol 2012;50:376-383.

15. Chandrashekar BS, Prabhakara S, Mohan T, et al. Characterization of Rubia cordifolia L. root extract and its evaluation of cardioprotective effect in Wistar rat model. Indian J Pharmacol 2018;50:-21.

16. Cai Y, Sun M, Xing J, Corke H. Antioxidant phenolic constituents in roots of Rheum officinale and Rubia cordifolia: Structure-radical scavenging activity relationships. J Agric Food Chem 2004;52:7884-7890.

17. Shen CH, Liu CT, Song XJ, et al. Evaluation of analgesic and anti-inflammatory activities of Rubia cordifolia L. by spectrum-effect relationships. J Chromatogr B Analyt Technol Biomed Life Sci 2018;1090:73-80.

18. Zheng Z, Li S, Zhong Y, et al. UPLC-QTOF-MS identification of the chemical constituents in rat plasma and urine after oral administration of Rubia cordifolia L. Extract. Molecules 2017;22:1327.

19. Wu Y, Jin F, Wang Y, et al. In vitro and in vivo inhibitory effects of 6-hydroxyrubiadin on lipopolysaccharideinduced inflammation. Immunopharmacol Immunotoxicol 2017;39:107-116.

20. Nam W, Kim SP, Nam SH, Friedman M. Structureantioxidative and anti-inflammatory activity relationships of purpurin and related anthraquinones in chemical and cell assays. Molecules 2017;22:265.

21. Basu S, Ghosh A, Hazra B. Evaluation of the antibacterial activity of Ventilago madraspatana Gaertn., Rubia cordifolia Linn. and Lantana camara Linn.: Isolation of emodin and physcion as active antibacterial agents. Phytother Res 2005;19:888-894. 
22. Gunasekara T, Radhika N, Ragunathan KK, et al. Determination of antimicrobial potential of five herbs used in ayurveda practices against Candida albicans, Candida parapsilosis and methicillin resistant Staphylococcus aureus. Anc Sci Life 2017;36:187-190.

23. Xu K, Wang P, Wang L, et al. Quinone derivatives from the genus Rubia and their bioactivities. Chem Biodivers 2014; 11:341-363.

24. Qiao YF, Wang SX, Wu LJ, et al. [Studies on antibacterial constituents from the roots of Rubia cordifolia L]. Yao Xue Xue Bao 1990;25:834-839.

25. Eglen SJ. A quick guide to teaching $\mathrm{R}$ programming to computational biology students. PLoS Comput Biol 2009; 5:e1000482.

26. Peterson CT, Sharma V, Uchitel S, et al. Prebiotic potential of herbal medicines used in digestive health and disease. J Altern Complement Med 2018;24:656-665.

27. Peterson CT, Sharma V, Iablokov SN, et al. 16S rRNA gene profiling and genome reconstruction reveal community metabolic interactions and prebiotic potential of medicinal herbs used in neurodegenerative disease and as nootropics. PLoS One 2019; 14:e0213869.

28. Peterson CT, Rodionov DA, Iablokov SN, et al. Prebiotic potential of culinary spices used to support digestion and bioabsorption. Evid Based Complement Alternat Med 2019;2019:8973704.

29. Wobbrock JO. The Aligned Rank Transform for nonparametric factorial analyses using only ANOVA procedures. In: Proceedings of the ACM Conference on Human Factors in Computing Systems, New York, USA, 2011, 143-146.

30. Searle S. The American Statistician Vol. 34. Washington: American Statistical Association, 1980:216-221.

31. Peterson CT, Vaughn AR, Sharma V, et al. Effects of turmeric and curcumin dietary supplementation on human gut microbiota: A Double-Blind, Randomized, PlaceboControlled Pilot Study. J Evid Based Integr Med 2018;23: $2515690 X 18790725$.

32. Castaner O, Goday A, Park YM, et al. The gut microbiome profile in obesity: A systematic review. Int $\mathbf{J}$ Endocrinol 2018;2018:4095789.
33. Tseng $\mathrm{CH}, \mathrm{Wu} \mathrm{CY}$. The gut microbiome in obesity. J Formos Med Assoc 2019;118 Suppl 1:S3-S9.

34. Yassour M, Lim MY, Yun HS, et al. Sub-clinical detection of gut microbial biomarkers of obesity and type 2 diabetes. Genome Med 2016;8:17.

35. Guo X, Li S, Zhang J, et al. Genome sequencing of 39 Akkermansia muciniphila isolates reveals its population structure, genomic and functional diverisity, and global distribution in mammalian gut microbiotas. BMC Genomics 2017;18:800.

36. Walker A, Pfitzner B, Harir M, et al. Sulfonolipids as novel metabolite markers of Alistipes and Odoribacter affected by high-fat diets. Sci Rep 2017;7:11047.

37. David LA, Maurice CF, Carmody RN, et al. Diet rapidly and reproducibly alters the human gut microbiome. Nature 2014;505:559-563.

Address correspondence to:

Christine T. Peterson, PhD

Department of Family Medicine and Public Health Center of Excellence for Research and Training in Integrative Health

University of California San Diego, School of Medicine 9500 Gilman Dr. \#0725

La Jolla, CA 92093

USA

E-mail: chpeterson@ucsd.edu

Raja K. Sivamani, MD, MS, AP Department of Dermatology

University of California Davis 3301 C Street, Suite 1400 Sacramento, CA 95816

USA

E-mail: raja.sivamani.md@gmail.com 